\title{
Performance Analysis of IEEE802.11ac DCF Enhancement for VHT with Frame Aggregation
}

\author{
https://doi.org/10.3991/ijes.v4i3.5970 \\ Z. Machrouh, A. Najid \\ National Institute of Posts and Telecommunication, Rabat, Morocco
}

\begin{abstract}
IEEE 802.11ac standard has brought several significant improvements compared to its predecessor IEEE 802.11n. It managed to break the Gigabits barrier with a combination of both refining older techniques and presenting new ones. The new enhancements such as channel bonding, beamforming, frames aggregation and finer modulation allow Wireless Local Area Networks (WLAN) the use of Very High Throughput (VHT). The physical layer (PHY) data rates are in the range of Gbps in the $5 \mathrm{GHz}$ band. But the variety of releases and options available for this standard has left many ambiguities regarding its real capabilities. The Medium Access Control layer (MAC) throughput is influenced by several factors, causing the MAC efficiency to decrease. In this paper we present a performance analysis in the VHT with frame aggregation for different access mechanisms, different channels and different modulation schemes.
\end{abstract}

Index Terms-DCF, Frame aggregation, MAC Throughput, RTS/CTS.

\section{INTRODUCTION}

In the past few years Wireless Local Area Networks (WLAN) have been widely deployed due to their mobility, flexibility and efficiency. In order to deal with the increasing number of users, several IEEE 802.11 standards evolved, such as IEEE $802.11 \mathrm{a} / \mathrm{b} / \mathrm{g}$ products that provide data rates up to $54 \mathrm{Mbps}$ and IEEE $802.11 \mathrm{n}$ products that can reach up to $600 \mathrm{Mbps}$. But with the addition of millions of wireless devices daily and the increasing demand on access to information all time and everywhere, IEEE $802.11 \mathrm{ac}$ has become the go to standard to bring users evident advancements.

For IEEE 802.11 standards the mandatory medium access mechanism is the Distributed Coordination Function (DCF), it can either be used exclusively or along with Point Coordination Function (PCF) which is an optional technique that best suits delay sensitive data transmissions [1].

Another mechanism, Transmit Opportunity (TXOP) was introduced in 2005 by the standard IEEE 802.11e.

IEEE 802.11ac use TXOP Sharing as an enhancement to the DCF where a station (STA) can transmit its frames freely as long as the limit of the TXOP is not surpassed. In this paper we limit our analysis to the DCF scheme under various modulations and coding as well as the effects of aggregation and the new VHT preambles format.

A considerable amount of research have been devoted to the performance of IEEE 802.11 systems and the DCF, either by means of analytical models or by means of simulation. The most acclaimed analysis is presented in [1], where Bianchi offers a Markov Chain bi-dimensional model to represent the performance of DCF in an IEEE
802.11 scenario. Several studies based on Bianchi's model ensued, in [2] a performance analysis of DCF in an IEEE 802.11 ac scenario was undertaken with the presence of Hidden Nodes (HN) to evaluate the system's normalized throughput for the basic access and the RTS/CTS scheme in an error prone channel. An analysis where transmission probability was taken into consideration is carried on in [3] to compare theoretical and simulation findings. Another interesting comparison was presented in [4], this model yields accurate results of throughput for both standards IEEE 802.11 ac and IEEE 802.11n in both error free channel and error prone channel. The importance of DCF made it possible to investigate both packet transmission techniques, the basic access and the RTS/CTS mechanism in MU-MIMO scenario. The impact of using RTS/CTS scheme in high data rates and with high modulation coding was examined in [5] it shows the saturation throughput and MAC efficiency, for the A-MPDU aggregation scheme it is proven that the MAC efficiency gets lower the higher the data rates are.

The remainder of this paper is outlined as follows in section II we review both mechanisms of DCF along with the PHY and MAC features of IEEE 802.11 ac.

In section III we define the concept of normalized throughput and we provide the analytical techniques to compute the performance. Section IV presents simulation results and performance analysis and section V summarizes this paper.

\section{PRELIMINARY}

In this section we briefly survey the PHY layer and the MAC layer enhancements. We however only focus on key aspects to help us understand what makes IEEE 802.11ac better. We also present an overview of the basic access scheme and the RTS/CTS scheme.

\section{A. IEEE802.11ac main features:}

Unlike the legacy standards, IEEE 802.11ac operates in the $5 \mathrm{GHz}$ band which remarkably reduces interferences and allows wider channels. Not only both $20 \mathrm{MHz}$ and $40 \mathrm{MHz}$ bands are supported, but IEEE 802.11ac support up to $160 \mathrm{MHz}$ band. Moving from $40 \mathrm{MHz}$ to $80 \mathrm{MHz}$ allows a $117 \%$ increase in data rates, while the leap towards $160 \mathrm{MHz}$ channels increases the data rates by $333 \%$ compared to a $40 \mathrm{MHz}$ channels [6]. Obtaining a $160 \mathrm{MHz}$ channel can be done by combining contiguous bands or non-contiguous bands. The use of more spatial stream is also an important feature, the first IEEE 802.11ac products to appear in the market supported 2 or 3 spatial streams which is the same as IEEE $802.11 \mathrm{n}$ but it is possible to use up to 8 spatial streams to provide transmissions at high data rates for multiple clients at the same time. 
Another enhancement is beamforming, IEEE 802.11n introduced multiple beamforming techniques and in order to provide the sought after performance both ends of the link had to share the same technique.

To overcome this inconveniency, IEEE 802.11ac adopted one method of beamforming the Null Data Packet (NDP) [7], [8] with this method the Beamformer can precisely steer each beam to the targeted receiver (Beamformee) and constructively use interferences.

IEEE $802.11 \mathrm{ac}$ also continue to expand complex modulation techniques, taking a leap towards 256 QAM with the optional coding rates $3 / 4$ and $5 / 6$. The basic set of rates is known as MCS0-9 it provides several increases in data rate as we can see completely detailed in [9]. Although 256 QAM present a 33\% efficiency gain over 64 QAM, in extended range transmission it is preferable to use lower QAM levels since benefits of higher modulation schemes prove to be minimal [10].

\begin{tabular}{|c|c|c|c|c|c|c|c|}
\hline \multirow{2}{*}{$\begin{array}{l}\text { HT mixed mode } \\
(802.11 \mathrm{n})\end{array}$} & $8 \mu \mathrm{s}$ & $\begin{array}{l}\text { nodulat } \\
8 \mu \mathrm{s}\end{array}$ & $4 \mu \mathrm{s}$ & $4 \mu \mathrm{s}$ & $\overline{4 \mu s}$ & $\begin{array}{c}\mathrm{HT} \text { modulation } \\
4 \mu \mathrm{s}\end{array}$ & Variable \\
\hline & L-STF & L-LTF & L-SIG & HT-STF & HT-LTF & HT-LTF & Data \\
\hline
\end{tabular}

When an IEEE802.11ac device transmits, legacy devices must be able to avoid transmitting for the length of time used on the medium. To meet this requirement, the format of the VHT physical layer frame is similar to the HT mixed-mode format used in IEEE802.11n as shown in Fig. 1 and it begins with the same fields as IEEE802.11a frames [8], but some subtle differences were also introduced to enable MU-MIMO transmissions.

For the MAC layer, IEEE 802.11 ac preserve many of the improvements that were first introduced in IEEE $802.11 \mathrm{n}$, such as frame aggregation schemes in which MSDUs share a common MAC header and are aggregated in an A-MSDU which is then encapsulated in an MPDU. MPDUs with different MAC headers are aggregated into an A-MPDU. IEEE 802.11ac increased the sizes of AMSDU to 11406 bytes and A-MPDU to 1048575 bytes and requires all frames to be transmitted as an A-MPDU even if there is only one MPDU. More details about the aggregation schemes and the MAC layer enhancements could be found in [5] and [10].

VHT mode (802.11ac)

\begin{tabular}{|c|c|c|c|c|c|c|c|}
\hline \multirow[b]{2}{*}{$8 \mu \mathrm{s}$} & \multicolumn{2}{|c|}{$\begin{array}{l}\text { OFDM PHY modulation } \\
\text { (identical for all users) }\end{array}$} & & & \multicolumn{2}{|c|}{$\begin{array}{l}\text { VHT modulation } \\
\text { (received per-user) }\end{array}$} & \multirow[b]{2}{*}{ Variable } \\
\hline & $8 \mu \mathrm{s}$ & $4 \mu \mathrm{s}$ & $8 \mu \mathrm{s}$ & $4 \mu \mathrm{s}$ & $8 \mu \mathrm{s}$ & $4 \mu \mathrm{s}$ & \\
\hline L-STF & L-LTF & L-SIG & VHT-SIG-A & $\begin{array}{l}\text { VHT- } \\
\text { STF }\end{array}$ & VHT-LTF & $\begin{array}{l}\text { VHT } \\
\text { SIG-B }\end{array}$ & Data \\
\hline
\end{tabular}

Figure 1. HT-mixed and VHT PPDU format

\section{B. IEEE 802.11ac Distributed Coordination Function}

For IEEE 802.11 standards, DCF is the fundamental medium access mechanism, it employs Carrier Sense Multiple Access with Collision Avoidance (CSMA/CA). Retransmission of collided packets is managed according to the Binary Exponential Backoff (BEB) algorithm to combat network congestion. At first the channel is monitored by a STA willing to transmit, when it is sensed idle for a period of time equal to a DCF InterFrame Space (DIFS) the transmission is carried on. The time following an idle DIFS is slotted and transmissions are only allowed at the beginning of a slot time $(\sigma)$. If the channel is sensed busy, the STA continue monitoring and generates a random backoff time in the range $[0, \mathrm{CW}]$, with $\mathrm{CW}$ being the contention window.

This range starts with a minimum value $C W_{\min }$ which doubles after each unsuccessful transmission until reaching $C W_{\text {max }}=2^{m} C W_{\text {min }}, \mathrm{m}$ stands for the maximum backoff stage. As long as the channel is sensed idle the backoff time counter is decremented, when detecting a transmission on the channel it is frozen and as soon as the channel becomes idle again it is reactivated, when it reaches zero the STA is allowed to transmit.

For IEEE $802.11 \mathrm{ac}$, the backoff procedure starts after a time period equal to a Short InterFrame Space (SIFS) if a Block Acknowledgement (BA) is not received by the STA after sending a Block Acknowledgement Request (BAR). DCF defines two techniques for transmission, the basic access scheme which is a two-way handshaking technique and the Request to Send/Clear to Send (RTS/CTS) which is a four-way handshaking technique. For the basic access the sender awaits the reception of a positive Acknowledgement frame to ensure the success of the transmission, while for the RTS/CTS scheme, the STA sends a RTS frame after the medium is sensed free for a DIFS period, if the receiver responds with a CTS frame after a SIFS period, the transmission starts according to the rules of the basic access scheme.

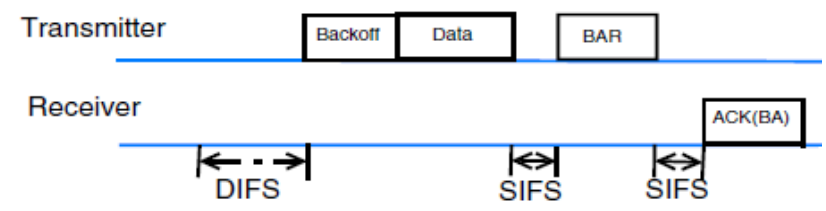

Figure 2. Basic Access Scheme

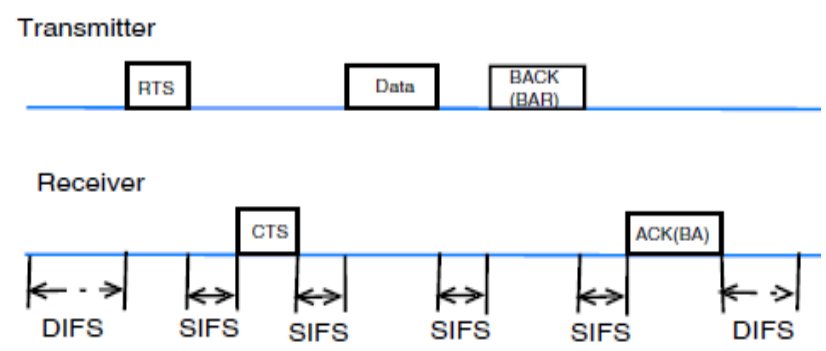

Figure 3. RTS/CTS Access Scheme 
The other STAs can benefit from the RTS and CTS frames to update their Network Allocation Vector (NAV) that contains information about the period of time in which the medium will remain busy.

Another benefit of using the RTS/CTS mechanism is that it provides a remedy for the Hidden Nodes problem. It also limits collision that can only occur on the RTS frames and if both stations employ RTS/CTS mechanism, it is detected by the absence of the CTS response.

\section{THROUGHPUT ANALYSIS}

In our analysis we operate in saturation mode, with a fixed number of stations, each one always having a packet available to transmit. Conforming to the Markov chain model from [1], we represent the DCF with the stochastic processes $b(t)$ and $s(t)$ that model the backoff time counter and the backoff stage respectively. This model also assumes that the collision probability is constant regardless of the retransmission suffered.

A random slot time is chosen where the STA senses the channel that can either be idle, busy transmitting or busy due to a collision. The STA attempts to transmit with probability $\tau$ given by:

$$
\tau=\frac{2(1-2 p)}{(1-2 p)\left(W_{0}+1\right)+p W_{0}\left(1-(2 p)^{m}\right)}
$$

Where $W_{0}=C W_{\text {min }}$. As we can see $\tau$ depends on the contention window size and the collision probability along with the maximum backoff stage $\mathrm{m}$. Collisions occur if one of the remaining $n-1$ STAs in the network transmit at the same time, $1-\tau$ is the probability that at least one STA is idle and $(1-\tau)^{n-1}$ is the probability that the remaining $n-1$ STAs are idle. Therefor we can express the collision probability as follows:

$$
\mathrm{p}=1-(1-\tau)^{\mathrm{n}-1}
$$

Through a fixed point iteration technique the nonlinear equations (1) and (2) can be solved.

The solutions enable us to represent the normalized system throughput $\mathrm{S}$, which is the ratio of the average payload size transmitted in a slot time over the length of the slot time.

$$
\mathrm{S}=\frac{P_{t r} P_{S} \mathrm{E}[\mathrm{P}]}{\mathrm{E}[\mathrm{T}]}
$$

$P_{t r}$ is the probability that at least one transmission has occurred in a slot time, $P_{S}$ is the probability that the transmission is successful, $\mathrm{E}[\mathrm{P}]$ is the average length of payload and $\mathrm{E}[\mathrm{T}]$ is the average slot time. Always conforming to the details in [1] we have:

$$
\begin{gathered}
P_{\text {tr }}=1-(1-\tau)^{n} \\
P_{S}=\frac{n \tau(1-\tau)^{n-1}}{1-(1-\tau)^{n}} \\
E[T]=\left(1-P_{\text {tr }}\right) \sigma+P_{\text {tr }} P_{S} T_{S}+P_{t r}\left(1-P_{S}\right) T_{C}
\end{gathered}
$$

$1-P_{t r}$ : Probability that the slot is empty.

$\mathrm{P}_{\text {tr }} \mathrm{P}_{\mathrm{S}}$ : Probability that there is a successful transmission.

$\mathrm{P}_{\mathrm{tr}}\left(1-\mathrm{P}_{\mathrm{S}}\right)$ : Probability that there is a collision.

$\sigma$ is the duration of an empty slot time, $T_{S}$ the period of time the channel is sensed busy due to a successful transmission and $T_{C}$ is the time the channel is busy because of collisions. The expressions of $T_{S}$ and $T_{C}$ differ according to the access mechanism employed. For the basic access we have:

$$
\mathrm{T}_{\mathrm{C}}^{\mathrm{b}}=\mathrm{T}_{\mathrm{S}}^{\mathrm{b}}=\mathrm{T}_{\mathrm{data}}+\text { DIFS }
$$

And for the RTS/CTS access mechanism we have:

$$
\begin{gathered}
\mathrm{T}_{\mathrm{C}}^{\mathrm{rts}}=\mathrm{T}_{\text {phy }}+\mathrm{T}_{\text {rts }}+\text { DIFS } \\
\mathrm{T}_{\mathrm{S}}^{\mathrm{rts}}=2 \mathrm{~T}_{\text {phy }}+\mathrm{T}_{\text {rts }}+2 \mathrm{SIFS}+\mathrm{T}_{\text {cts }}+\mathrm{T}_{\text {data }}+\text { DIFS }
\end{gathered}
$$

$T_{\text {data }}$ is the period of time in which data and Block Acknowledgement Request (BAR) frames are transmitted as well as Block Acknowledgement (BA) frames are received.

$\mathrm{T}_{\text {data }}=2 \mathrm{SIFS}+\mathrm{DIFS}+T_{B A R}+T_{B A}+\mathrm{T}_{\text {phy }}+\mathrm{T}_{\text {sym }} \mathrm{N}_{\text {sym }}(9)$

$\mathrm{T}_{\text {phy }}$ is detailed in (10) for the HT-mixed format and the VHT format. $T_{\text {sym }}$ is the symbol duration, it is determined by the bandwidth and the guard interval used. $N_{\text {sym }}$ detailed in (11) is the number of symbols needed to transmit the data, a key element in the expression of $N_{\text {sym }}$ is $N_{D B P S}$ it depends on the bandwidth, the selected modulation, the coding rate, the type of aggregation and the number of spatial streams. An example of calculating $N_{D B P S}$ and $T_{\text {sym }}$ is detailed in [11] and more details can be found in [4].

\section{PERFORMANCE ANALYSIS}

In our paper we first analyze IEEE 802.11ac throughput for different values of the transmission probability and error probability. Then we analyze the network performance under various modulation and coding rates with different channel bandwidths and different types of frame aggregation. We choose to validate our simulator a BSS with a single AP and various contending STAs, in all these simulations we conform to the parameters set in Table I, using the IEEE TGac specifications [12].

\section{A. Transmission probability and error probability:}

As seen in section III, $\tau$ depends on the size of the network and the parameters $m$ and $\mathrm{W}$ which are a PHY layer set parameters and cannot be tuned. The throughput suffers in certain scenarios due to the lack of flexibility in adapting these parameters, we can also deduce that optimal performance can be achieved for every network scenario for the right transmission probability value. Fig.4 shows the effect of the transmission probability on the system theoretical throughput. The results of this simulation have been obtained using the system parameters reported in Table. 1 as we can see from the figure, the slightest difference in the estimated value of $\tau$ leads to remarkable changes in the throughput values. 


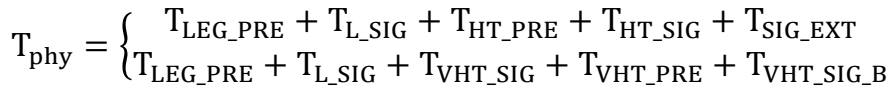

$$
\begin{aligned}
& N_{\text {sym }}=\left\{\begin{array}{c}
\mathrm{m}_{\mathrm{STBC}}\left[\frac{8 \mathrm{~N}_{\mathrm{A}-\mathrm{MSDU}}\left(\mathrm{L}_{\mathrm{PLD}}+\mathrm{L}_{\mathrm{FA}-\mathrm{OH}}+\mathrm{L}_{\mathrm{MAChdr}}\right)+\mathrm{L}_{\mathrm{SER}}+\mathrm{L}_{\mathrm{TAIL}} \mathrm{N}_{\mathrm{ES}}}{\mathrm{m}_{\mathrm{STBC}} \mathrm{N}_{\mathrm{DBPS}}}\right], \text { for A - MSDU aggregation } \\
\mathrm{m}_{\mathrm{STBC}}\left[\frac{8 \mathrm{~N}_{\mathrm{A}-\mathrm{MPDU}}\left(\mathrm{L}_{\mathrm{PLD}}+\mathrm{L}_{\mathrm{FA}-\mathrm{OH}}+\mathrm{L}_{\mathrm{MAChdr}}\right)+\mathrm{L}_{\mathrm{SER}}+\mathrm{L}_{\mathrm{TAIL}} \mathrm{N}_{\mathrm{ES}}}{\mathrm{m}_{\mathrm{STBC}} \mathrm{N}_{\mathrm{DBPS}}}\right], \text { for A - MPDU aggregation } \\
\mathrm{m}_{\mathrm{STBC}}\left[\frac{8 \mathrm{~N}_{\mathrm{A}-\mathrm{MPDU}}\left(\mathrm{L}_{\mathrm{PLD}} \mathrm{N}_{\mathrm{A}-\mathrm{MSDU}}+\mathrm{L}_{\mathrm{FA}-\mathrm{OH}}+\mathrm{L}_{\mathrm{MAChdr}}\right)+\mathrm{L}_{\mathrm{SER}}+\mathrm{L}_{\mathrm{TAIL}} \mathrm{N}_{\mathrm{ES}}}{\mathrm{m}_{\mathrm{STBC}} \mathrm{N}_{\mathrm{DBPS}}}\right], \text { for hybrid aggregation }
\end{array}\right.
\end{aligned}
$$

As the number of stations increase, we can see that the throughput is more sensitive to the variation in the values of $\tau$ and there is less dependency on the system parameters.

As for Fig. 5 we investigate the impact of the channel errors, more specifically the effect of the conditional collision probability on the throughput. Unlike the transmission probability, the number of stations in the network does not affect the throughput it is rather due to the increase of the possibility that transmitted packets will collide. Since we are conforming to the key assumption of constant independent collision probability.

\section{B. Normalized throughput vs. packet size:}

To investigate the performance of both access mechanisms of DCF, we choose a scenario with one AP and various contending STAs.

We plot the uplink throughput of the whole BSS for the three aggregation types while using different primary channels. Fig.6 and Fig. 7 show the system throughput for the basic access scheme and the RTC/CTS scheme.

A-MSDU frame enables to transmit more MSDUs, thus improving the ratio between the number of data bits transmitted to the MAC and PHY layers overhead. But although it has the least overhead it is limited in terms of the number of aggregated frames per PHY overhead.

A-MPDU aggregation can aggregate more frames per PHY overhead, still it does not achieve the best performance mainly due to the fact that it is more prone to channel errors; the entire A-MSDU needs to be retransmitted if any error occur during transmission.

The hybrid aggregation achieves remarkably the best performances, due to the reduction in MAC overheads which gets better with the increase of A-MSDU size.

In Fig. 8 both access mechanisms are evaluated for 40 , 80 and $160 \mathrm{MHz}$, for top modulation and coding rates for the same BSS and the same parameters. Qualitatively stating the RTS/CTS mechanism is more effective when the packet size increases. Its performance is very similar under several PHY layer changes due to the fact that it loosely depend on the network size and the contention window. It is also easily achievable to determine a threshold for the packet size over which we can switch from the basic access to the RTS/CTS scheme. Still aggregation proves to be better even for other PHY parameters or other scenarios.

\section{CONCLUSION}

In this paper, we presented a simple analytical model to study the effect both access mechanisms of the Distributed Coordination Function, have on the IEEE 802.11 ac throughput with and without aggregation. Using the proposed model, we evaluated the impact of the
TABLE I. SIMULATION PARAMETERS

\begin{tabular}{|l|l|}
\hline \multicolumn{1}{|c|}{ Parameter } & \multicolumn{1}{c|}{ Value } \\
\hline Slot time $\sigma$ & $9 \mu \mathrm{s}$ \\
\hline SIFS & $16 \mu \mathrm{s}$ \\
\hline DIFS & $34 \mu \mathrm{s}$ \\
\hline RTS frame size $L_{r t s}$ & 20 bytes \\
\hline CTS frame size $L_{c t s}$ & 14 bytes \\
\hline MAC compressed BAR frame size $L_{B A R}$ & 24 bytes \\
\hline MAC compressed BA frame size & 32 bytes \\
\hline MAC overhead $L_{M A C h d r}$ & 34 bytes \\
\hline Minimum contention window $C W_{\min }$ & 15 \\
\hline Length of service bits $L_{S E R}$ & 16 bits \\
\hline Length of tail bits $L_{T A I L}$ & $6 . N_{E S}$ bits \\
\hline Number of encode stream $N_{E S}$ & 2 \\
\hline
\end{tabular}

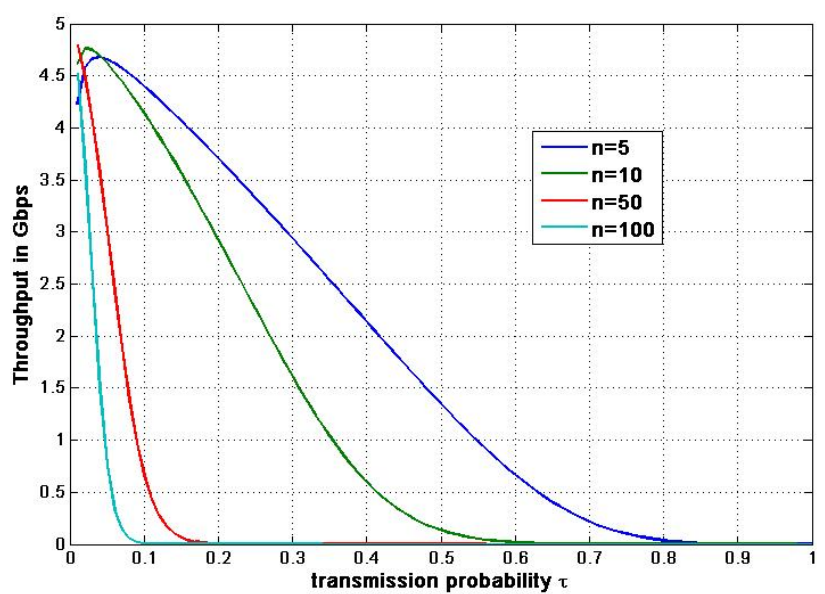

Figure 4. Throughput vs. Transmission probability

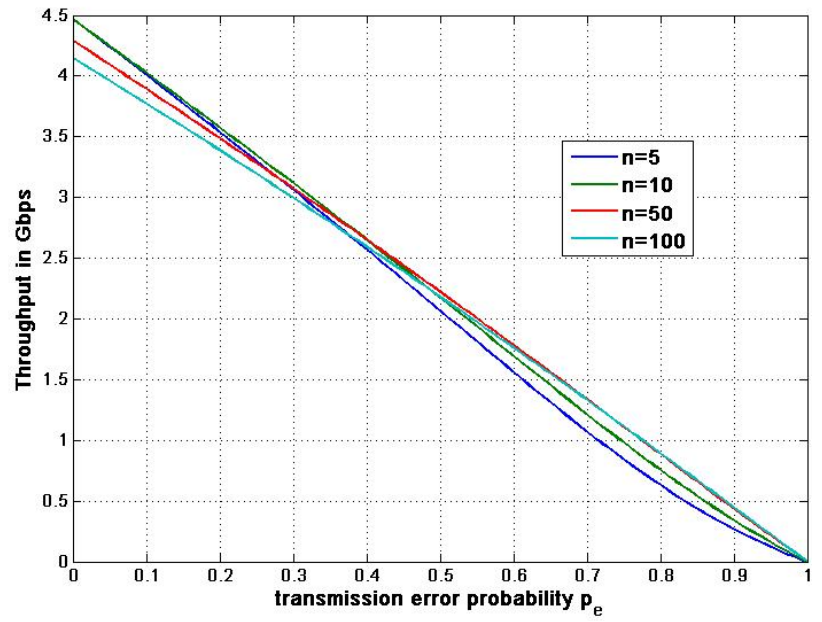

Figure 5. Throughput vs. Transmission error probability 


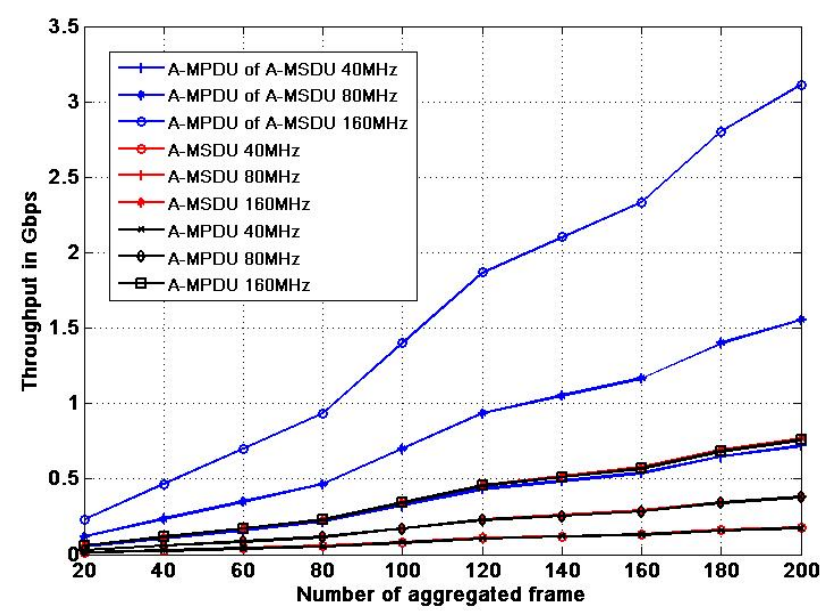

Figure 6. Throughput for the basic access scheme

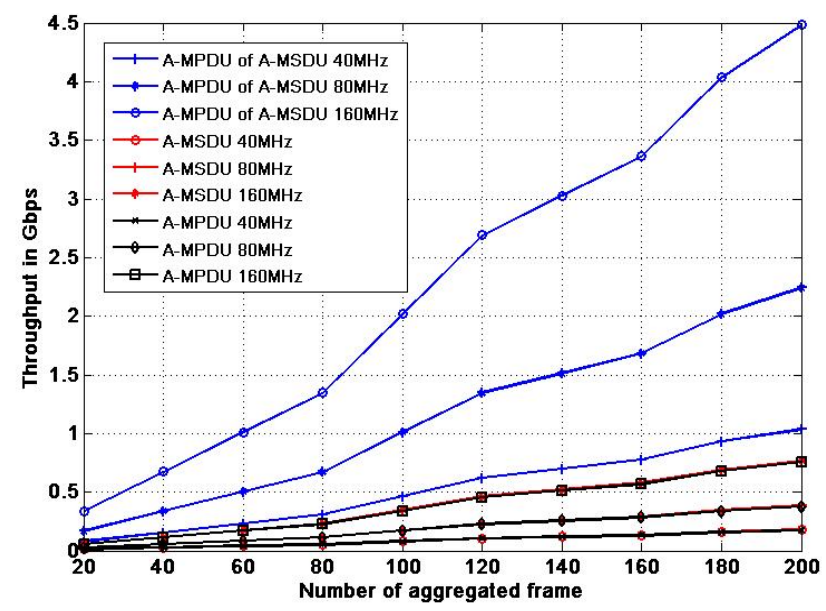

Figure 7. Throughput for the RTS/CTS access scheme

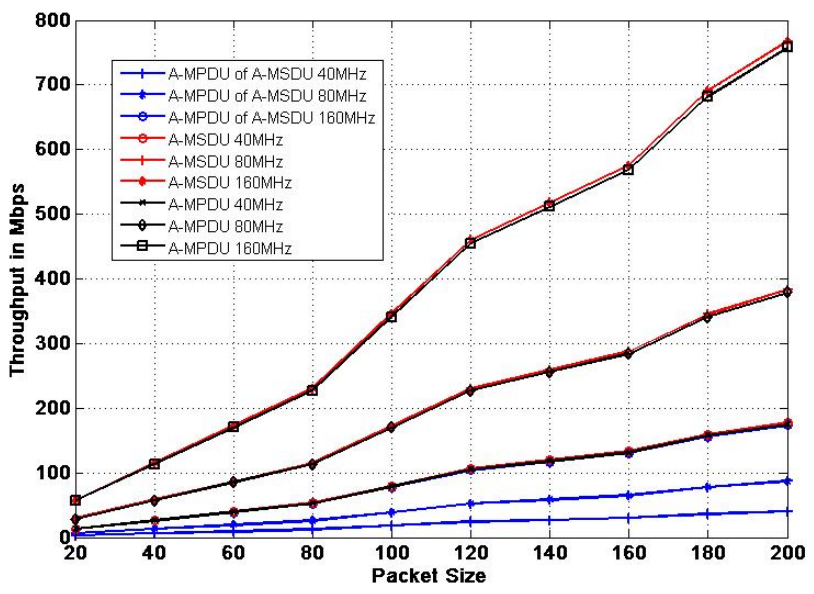

Figure 8. Throughput against the packet size

RTS/CTS and the basic access schemes in VHT networks. We have also shown the effects of the transmission probability and the transmission error probability on the system throughput. Although wider channel bandwidth and new modulations scheme are offered in the IEEE 802.11ac standards, the throughput can still be optimized to get to full potential. For further work, we planned to investigate the effectiveness of TXOP in MUMIMO transmissions and the effects of the PHY frame format enhancements brought to the throughput optimization.

\section{ACKNOWLEDGMENT}

This work was sponsored by the National Center for Technical and Scientific Research (CNRST) in Morocco.

\section{REFERENCES}

[1] G.Bianchi, "Performance Analysis of the IEEE 802.11 Distributed Coordination Function,"IEEE Journal on Selected Areas in Communications, Vol.18, No.3, pp.535-547.2000.

[2] Zheng Chang, Olli Alanen, Toni Huovinen, Timo Nihtilä, Eng Hwee Ong, Jarkko Kneckt and Tapani Ristaniemi, "Performance Analysis of IEEE 802.11ac DCF with Hidden Nodes," 978-14673-0990-5/12/\$31.00 (C)2012 IEEE.

[3] Gul Zameen Khan, Ruben Gonzalez, Eun-Chan Park, "A Performance Analysis of MAC and PHY Layers in IEEE 802.11ac Wireless Network", Jan. 31 Feb. 3, 2016 ICACT2016.

[4] Oran Sharon, Yaron Alpert, "MAC level Throughput comparison: 802.11 ac vs.802.11n“, Physical Communication. September 2014. http://dx.doi.org/10.1016/j.phycom.2014.01.007

[5] Ha Cheol Lee, "A Performance of MAC Layer Over Error-prone Channel in The IEEE 802.11ac", Proc. of the Second Intl. Conf. on Advances In Computing, Electronics and Communication, 2014.

[6] Xirrus White Paper,"802.11ac Demystified", Xirrus Inc.2013.

[7] Aruba White Paper," 802.11ac In-Depth,” (C) 2012 Aruba Networks, Inc.

[8] Matthew S. Gast, "802.11ac: A Survival Guide," O'Reilly Media, Inc. isbn=9781449343149.

[9] Ha Cheol Lee, "DCF Throughput Analysis of IEEE 802.11a/g/nbased Mobile LAN over Correlated Fading Channel", International Journal on Electrical Engineering and Informatics - Volume 3, Number 4, 2011.

[10] Ruckus white paper,"802.11ac Next Steps to Next Generation Wi-Fi”, October 2014.

[11] Ruizhi Liao, Boris Bellalta, Jaume Barcelo, Victor Valls and Miquel Oliver, "Performance analysis of IEEE 802.11ac wireless backhaul networks in saturated conditions," EURASIP Journal on Wireless Communications and Networking 2013.

[12] IEEE P802.11 Wireless LANs Specification Framework for TGac. January 2011.

\section{AUTHORS}

Zineb MACHROUH has Master degrees in Electronics and Telecommunications and is currently preparing her $\mathrm{PhD}$ thesis in Wireless Sensor Networks at National Institut of Postes and Telecommunication in Morocco.

Abdellah NAJID has Master degrees in Networking and Communication Systems and $\mathrm{PhD}$ thesis in Microwaves and Wireless Networking in 1997 from Institut National Polytechnique de Toulouse INPT France. Abdellah NAJID was working as researcher Engineer at INRIA Labs from 1998 to 2000. Abdellah NAJID research fields are routing Protocols, wireless networking,Microwave and Antennas design. Actually, Abdellah NAJID is full Professor at National Institut of Postes and Telecommunication.

Submitted 20 June 2016. Published as resubmitted by the authors 17 September 2016. 